Article

\title{
A Haptic Model for the Quantum Phase of Fermions and Bosons in Hilbert Space Based on Knot Theory
}

\author{
Stefan Heusler ${ }^{+}\left(\mathbb{C}^{-}\right.$and Malte Ubben $*,+(-)$ \\ Institut für Didaktik der Physik, Universität Münster, 48149 Münster, Germany; sheus_01@uni-muenster.de \\ * Correspondence: malte.ubben@uni-muenster.de; Tel.: +49-0251-833-9471 \\ † Current address: Wilhelm-Klemm-Str. 10, 48149 Münster, Germany.
}

Received: 19 December 2018; Accepted: 7 March 2019; Published: date

\begin{abstract}
A generalization of the famous Dirac belt trick opens up the way to a haptic model for quantum phases of fermions and bosons in Hilbert space based on knot theory. We introduce a simple paper strip model as an aid for visualization of the quantum phases before and after Hopf-mapping, which can be extended to arbitrary spin states with almost no mathematical formalism. Knot theory arises naturally, leading to the Jones polynomials derived from Artin's braid group for fermionic knots and for bosonic links. The paper strip model explicitly illuminates the relation between these knots and links within the $S U(2)$-representation of spin-jstates in $C^{2 j+1}$ before Hopf-mapping and the number $p=2 j$ of nodes in the stellar representation in $C P^{1}$ after Hopf mapping.
\end{abstract}

Keywords: knot theory; spin-statistics; haptic model; boson-fermion correspondence

\section{Introduction}

The relation between quantum states in Hilbert space and observables is highly non-trivial.

As an illustrative example, we consider the case of spin $\frac{1}{2}$. Using the unitary operator $U \in S U(2)$, we can reach all pure spin states from the "spin up" state $|0\rangle$ as an orbit $U|0\rangle=u|0\rangle+v|1\rangle$, with complex amplitudes $(u, v) \in C^{2}$ fulfilling $|u|^{2}+|v|^{2}=1$. Geometrically, all these spin states form the hypersphere $S_{3}$ in four dimensions. Thus, seen as a manifold, the complex rotation group $S U(2)$ is isomorphic to $S_{3}$. It is well known that the periodicity of spin- $\frac{1}{2}$ states in $S_{3}$ is $(4 \pi)$ rather than $(2 \pi)$, meaning that after a $(2 \pi)$-rotation, the spinor changes its sign, and only after a $(4 \pi)$-rotation, the spinor is mapped onto itself. Dirac proposed a geometric interpretation of spin- $\frac{1}{2}$ states in $C^{2}$, the so-called "Dirac belt" [1]. After Hopf-mapping, the $S U(2)$-operation $U$ is mapped to a rotation $S O(3)$ in three dimensions due to $S O(3) \simeq S U(2) / Z_{2}$. For the Hopf-mapping, the two-to-one mapping $Z_{2}$ is essential, which identifies the spinor with a positive and with a negative sign. After this identification, the orbit of the state $|0\rangle$ can be represented on the Bloch sphere $S_{2}$, with a periodicity of $(2 \pi)$. Due to this mapping, the Dirac belt is related to a Möbius strip [2]. It is important to distinguish the geometry of $S_{3}$ carefully, which allows for a $(4 \pi)$-periodicity, and the description after Hopf-mapping to $S_{2}$, where the usual $(2 \pi)$-periodicity emerges. In order to emphasize this difference, we will call the sphere $S_{3}$ the " $(4 \pi)$-realm" and the sphere $S_{2}$ the " $(2 \pi)$-realm". All observables are part of the $(2 \pi)$-realm. Thus, in order to understand the relation between quantum states in Hilbert space and observables, it is important to understand the (generalized) Hopf-mapping $S_{3} \rightarrow S_{2}$ from the $(4 \pi)$-realm to the $(2 \pi)$-realm. In the present paper, we generalize the Dirac belt in Hilbert space to arbitrary spin states, and show how the mapping from the $(4 \pi)$-realm to the $(2 \pi)$-realm can be encoded in a simple haptic model, which we denote as the paper strip model.

In Section 2, we discuss the simplest possible model, that is representations of the group $U(1)$ in the $(2 \pi)$-realm. In Section 3, based on Artin's braid group, a close relation between the phase changes upon rotation of fermionic and bosonic states in Hilbert space is derived, leading to explicit formulas 
for the corresponding Jones polynomials. In Section 4, we generalize the construction of the Dirac belt to all spin states in the $(4 \pi)$-realm, revealing an explicit mapping from fermionic knots and bosonic links of $S U(2)$-representations in Hilbert space to observable nodes in wave functions, e.g., in the stellar representation. Possible applications are mentioned in the summary and outlook (Section 7).

\section{Great Circles and Double Windings in the $(2 \pi)$-Realm}

Our discussion begins with the simplest possible geometric model-that of a circle $S_{1}$ in ordinary $R^{3}$-space-as the most prominent example for the $(2 \pi)$-realm. Consider a differentiable function $g(\phi)$ on $S_{1}$. The rotation by an angle $\theta$ can be described by the Taylor series:

$$
\exp \left[i \theta \frac{1}{i} \partial_{\phi}\right] g(\phi)=g(\phi+\theta)
$$

Thus, $\frac{1}{i} \partial_{\phi}$ is the generator of rotations on $S_{1}$. We search for a spectral decomposition of the rotation operator. We can decompose the function $g(\phi)$ in its Fourier components as $g(\phi)=\sum_{m \in Z} a_{m} \exp [i m \phi]$. The spectral decomposition of the generator of rotations is then given by the diagonal matrix:

$$
\begin{aligned}
d_{k p}=\left\langle k\left|\frac{1}{i} \partial_{\phi}\right| p\right\rangle & \equiv \\
\frac{1}{2 \pi} \int_{0}^{2 \pi} \exp [-i k \phi] \frac{1}{i} \partial_{\phi} \exp [+i p \phi] & =\delta_{k p} p .
\end{aligned}
$$

We introduce a haptic model for the matrix $d_{p p} \propto(\ldots,-2,-1,0,1,2, \ldots)$ using a simple paper strip. The complex phase $\exp [+i p \phi]$ is winding $2 p$ times around the circle $S_{1}$. In our model, the paper strip of "length" $2 \pi$ has to be twisted $2 p$-times clockwise (R: rightwards $k>0$ ) or counter-clockwise (L: leftwards $k<0$ ). Afterwards, the starting point $\phi=0$ and the end point $\phi=2 \pi$ are glued together to close the circle. In terms of the homotopy group, this decomposition into Fourier modes just corresponds to $\pi_{1}\left(S_{1}\right)=Z$. This simple model can now be generalized to great circles in $D \geq 4$ dimensions. Here, the $2 \pi$-periodicity is extended to a $4 \pi$-periodicity. For the modes $d_{k k}$, at first sight, nothing should change beside the fact that the paper strip is traversed twice. However, more modes emerge, since:

$$
\begin{aligned}
d_{k p}=\left\langle\frac{k}{2}\left|\frac{1}{i} \partial_{\phi}\right| \frac{p}{2}\right\rangle & \equiv \\
\frac{1}{4 \pi} \int_{0}^{4 \pi} \exp \left[-i \frac{k}{2} \phi\right] \frac{1}{i} \partial_{\phi} \exp \left[+i \frac{p}{2} \phi\right] & =\delta_{k p} \frac{p}{2}
\end{aligned}
$$

leads to modes with "half-integer" windings. In the paper strip model, beyond modes with an even number of twists, those with an odd number of twists emerge, where the complex phase $\exp [+i(p / 2) \phi]$ is winding $p$ times around the circle $S_{1}$. As shown in Figure 1, gluing together the "start point" $\phi=0$ and the "end point" $\phi=2 \pi$ leads to those new modes with an odd number of twists to Möbius strips with only one surface. Based on this simple model, many characteristics of elementary particles can be modeled [3]. 


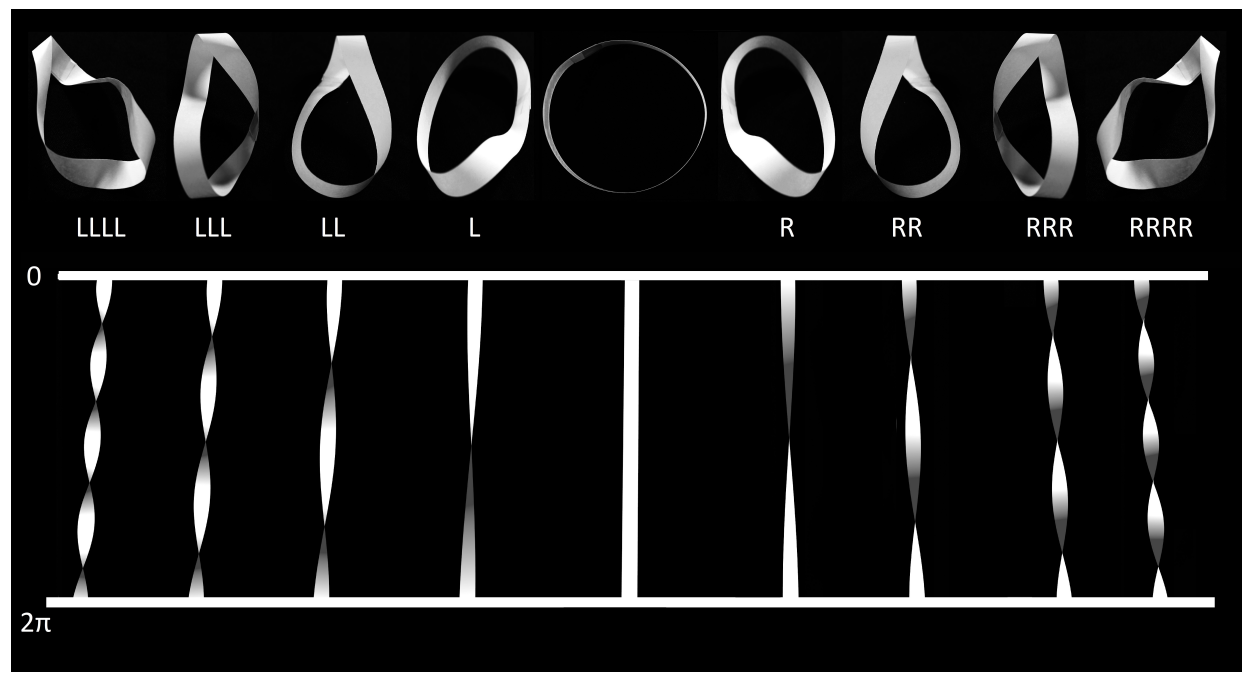

Figure 1. The paper strip model for the phase factor $\exp [+i(p / 2) \phi]$ : An odd number of twists $(|p|=1,3)$ leads to a Möbius strip topology of the paper strip with only one surface. On a Möbius strip, after a rotation of $2 \pi$, the original position is reached upside-down. The modes with an even number of twists $(|p|=0,2,4)$ have two surfaces. A rotation of $2 \pi$ leads to the original position on the paper strip. Right-moving twists (R) emerge for $p>0$, while left-moving twists (L) have $p<0$.

\section{Jones Polynomials of Bosonic and Fermionic States in the $(4 \pi)$-Realm}

The three-sphere $S_{3}$ can be defined using four real parameters $(X, Y, Z, U)$ as:

$$
X^{2}+Y^{2}+Z^{2}+U^{2}=1
$$

In what follows, $(p, 2)$-torus knots turn out to be crucial for the generalization of the Dirac belt to the case of general spin $j=p / 2$. It is well known that knots and links can be described in terms of algebraic curves [4,5]. Consider the holomorphic function $C^{2} \rightarrow C$ given by $g(u, w)=u^{p}+w^{2}$. The intersection of the set of solutions of $g(u, v)=0$ with the three-sphere $S_{3}$ describes the $(p, 2)$-torus knot. Using a stereographic projection $S_{3} \rightarrow R^{3}$, it is possible to visualize the $(p, 2)$-torus knots emerging in $S_{3}$ projected to $R^{3}$ as:

$$
(X, Y, Z, U) \rightarrow\left(\lambda_{1}, \lambda_{2}, \lambda_{3}\right)=\left(\frac{X}{(1-U)}, \frac{Y}{(1-U)}, \frac{Z}{(1-U)}\right) .
$$

Note that the north pole $U=1$ has to be added as a point "at infinity". It is well known that the geodesic flow leaves tori of revolution invariant [6]. Explicitly, we may consider for example the parameterization:

$$
\left(\sqrt{\lambda_{1}^{2}+\lambda_{2}^{2}}-a\right)^{2}+\lambda_{3}^{2}=r^{2}
$$

We parameterize a general $(p, q)$-torus knot embedded in $R^{3}$ as:

$$
\begin{aligned}
\lambda_{1}^{j} & =(a+r \cos [p t]) \cos [q t] \\
\lambda_{2}^{j} & =(a+r \cos [p t]) \sin [q t] \\
\lambda_{3}^{j} & =r \sin [p t]
\end{aligned}
$$

Similar to Avrin [3], we consider the $(p, 2)$-torus knots for the spin $j$ state by selecting $p=2 j, q=2$. The simple paper strip model in the $(2 \pi)$-realm introduced in the last section naturally leads to knot theory. Indeed, the two edges of the paper strip then correspond to the principal bundle, leading either to a connected knot in $R^{3}$ (equivalently, in $S_{3}$ ) for half-integer $j$ after a $4 \pi$-rotation in $\eta \equiv q t=2 t$ or to a 
disconnected link for integer $j$ after a $2 \pi$-rotation in $\eta$ for two curves $r_{1}>r_{2}$ describing the two strands at the boundary of the paper strip. For integer $j$, the link is part of what we call the $(2 \pi) \times(2 \pi)$-realm, since each of the two geodesics of the link have a period of $(2 \pi)$.

A famous theorem of Alexander [7] states that every knot or link can be represented as a closed braid. Thus, the knot structure of fermionic and bosonic states can be constructed using Artin's braid group. For the derivation of Jones polynomials $J^{j}(t)$ characterizing the type of knot as a function of a phase variable $t$ for the homotopy of the highest weight state $|j, j\rangle$, Artin's braid group describing crossings between the two strands as shown in Figure 2 is sufficient. We can derive a recursion relation from the definition of the Jones polynomial, as shown in Figure 2. The result is:

$$
t^{-1} J^{j+1 / 2}(t)-t J^{j-1 / 2}(t)=\left(t^{1 / 2}-t^{-1 / 2}\right) J^{j}(t),
$$

leading for fermionic knots with $j \neq 1 / 2$ and for bosonic links with $j \neq 0$ to the explicit expression:

$$
J^{j}(t)=(-1)^{2 j+1}\left(t^{\frac{2 j-1}{2}}+\sum_{k=1}^{2 j-1}(-1)^{k+1} t^{k+\frac{2 j+1}{2}}\right)
$$

for integer and half-integer $j \geq 1$. For $j=0$, the Jones polynomial is given by $J^{j=l=0}(t)=-t^{-1 / 2}-t^{1 / 2}$; for spin $j=1 / 2$, the Jones polynomial is trivial, $J^{j=+1 / 2}(t)=1$. At first sight, for bosons, the distinction between the $(2 \pi)$-realm and the $(2 \pi) \times(2 \pi)$-realm (equivalently, the distinction between $S U(2) / Z_{2}$ operations and $S U(2)$ operations) seems irrelevant, since the behavior for observables is not changed due to the mapping. However, the close relation between bosons and fermions can only be understood when the full quantum state in Hilbert space is considered. Indeed, the links describing the bosonic homotopy and the knots describing the fermionic homotopy are intimately related, since the bosonic state $|l, l\rangle$ with integer $l$ in the $(2 \pi) \times(2 \pi)$-realm becomes the state $j=l \pm 1 / 2$ in the $(4 \pi)$-realm just by a local change of crossings between the two strands, as shown in Figure 2. In such a way, the origin of the boson-fermion correspondence, which is mathematically related to the Jacobi triple product identity [8], can be traced back to knot theory.
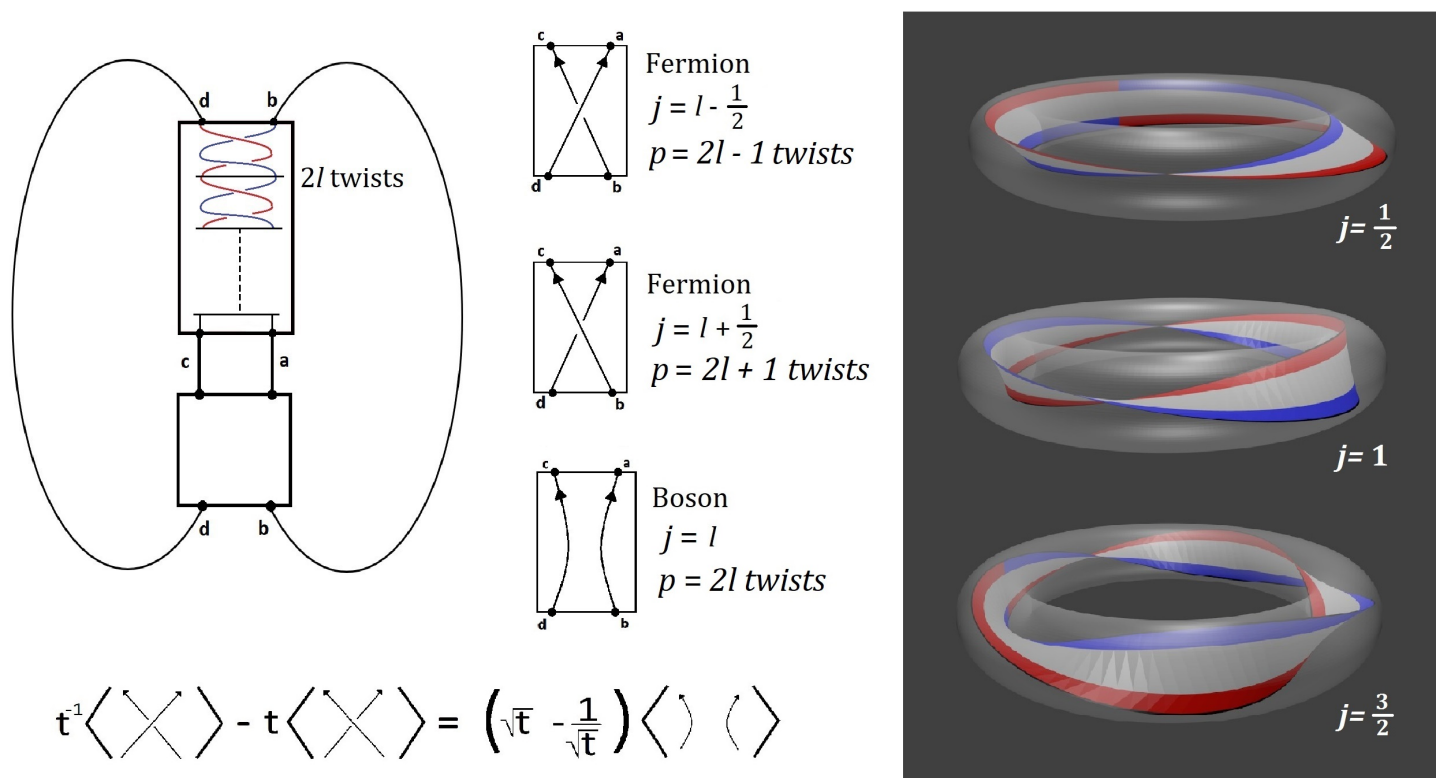

Figure 2. On the left: Derivation of the recursive formula for the Jones polynomials for the highest weight states $|j, \pm j\rangle$. The general spin states $j=\{l-1 / 2, l, l+1 / 2\}$ (with integer spin $l$ ) are obtained from the braid with $2 l$ twists by insertion of one of the three boxes, respectively. On the right: Paper strips with $p=1,2,3$ twists (R, RR, RRR) embedded in a torus, corresponding to $\operatorname{spin} j=1 / 2,1,3 / 2$, respectively. The red and blue edges of the paper strip correspond to the braids. 
Each knot corresponding to the highest-weight state $|j, j\rangle$ can be mirrored, which in the strip model is achieved by replacing $R$ twists with $L$ twists in the $(2 \pi)$-realm. The corresponding Jones polynomials for $|j,-j\rangle$ are obtained by the mapping $t \rightarrow t^{-1}$.

\section{A Generalization of the "Dirac Belt" Trick in the $(4 \pi)$-Realm}

Our starting point will be the case $j=\frac{1}{2}$. Following Dirac, we introduce a "belt", which is nothing but our paper strip extended from "length" $2 \pi$ to $4 \pi$ [1]. The two sides of the belt may be associated with two complex variables $(u, v) \in C^{2}$. We introduce the complex homogeneous coordinates $\left(Z_{1}, Z_{2}\right)$ as:

$$
\begin{aligned}
& Z_{1} \equiv u=e^{i \frac{\tau}{2}+i \frac{\phi}{2}} \cos \left[\frac{\theta}{2}\right] \\
& Z_{2} \equiv v=e^{i \frac{\tau}{2}-i \frac{\phi}{2}} \sin \left[\frac{\theta}{2}\right] .
\end{aligned}
$$

A little calculation (The coordinates $\lambda_{i}$ are projected back to $S_{3}$ as $X=\frac{\lambda_{1}}{\rho a}, Y=\frac{\lambda_{2}}{\rho a}, Z=\frac{\lambda_{3}}{\rho a}, U=$ $1-\frac{1}{\rho a}$ with $\rho=\sqrt{\lambda_{1}^{2}+\lambda_{2}^{2}}$. For $u=X+i Y$, we find $a=\frac{1}{\cos [\theta / 2]}$ for $r^{2}=a^{2}-1>0$.) confirms $1 / a=\cos [\theta / 2]$ for the tori defined in Equation (7) for the subset fulfilling $r^{2}=a^{2}-1$.

With a general quaternion $q$, a unitary operator $U_{q} \in S U(2)$ acting on the qubit state $|Q\rangle=$ $u|1 / 2,1 / 2\rangle+v|1 / 2,-1 / 2\rangle$ is given by:

$$
U_{q}(\alpha)=\exp [(\alpha / 2) q]=\cos [\alpha / 2]+q \sin [\alpha / 2] .
$$

A basis $I, J, K$ of the quaternions is given by $I \equiv-i \sigma_{1}, J \equiv-i \sigma_{2}, K \equiv-i \sigma_{3}$, where $\sigma_{i}$ are the Pauli matrices. Crucially, operations acting on the qubit state $|Q\rangle$ can be translated into motions of the Dirac belt describing the phase change, as shown in Figure 3.
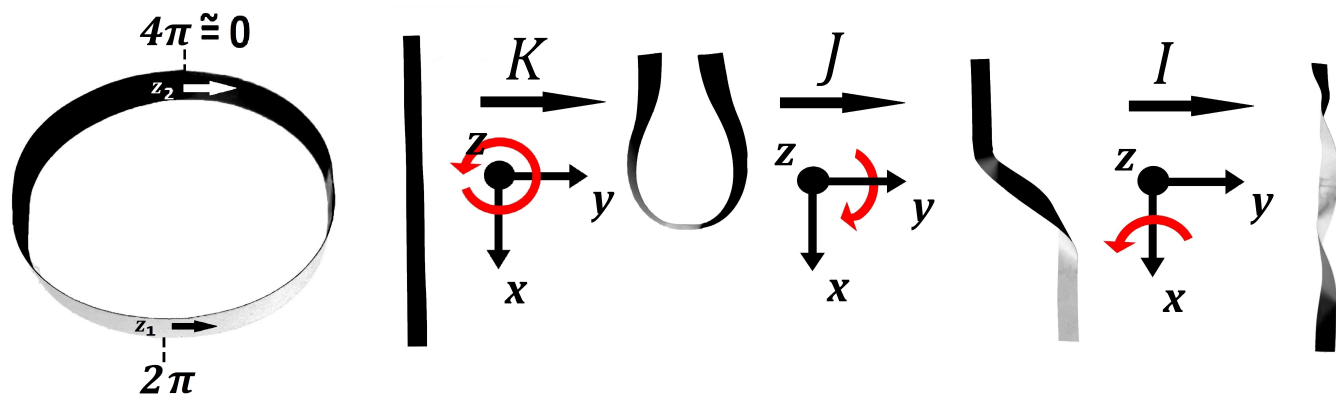

Figure 3. In the $(4 \pi)$-realm of $S U(2)$, the paper strip describing the quantum phase has a length of $(4 \pi)$ and always has two surfaces. In other words, in contrast to the $(2 \pi)$-realm, no double-valuedness of the wave function can emerge. Inner twists can be performed in three different "directions", which can be described using the quaternions $I \equiv-i \sigma_{1}, J \equiv-i \sigma_{2}$ and $K \equiv-i \sigma_{3}$. The non-commutativity is reproduced by the motion of the paper strip in the $(4 \pi)$-realm shown here, since the order of operations matters for the result. In particular, $I J K|*\rangle=-1|*\rangle$ for an arbitrary initial state $|*\rangle$.

It was first pointed out by P. Dirac that $(-1)$ can be represented equivalently by a double-twist rotating clockwise or counter-clockwise (Figure 4). Four twists lead back to the identity operator, $I^{4}=+1$; therefore, $I^{2}=I^{-2}=-1$. In our model, this is achieved by cutting the "Dirac belt" open, stretching it out, and pulling one end through the emerging loop, so that the loop becomes a mirrored version of itself, before closing it again "the other way round". This operation changes the number of twists in the $(4 \pi)$-realm by four. For a single complex variable in the $(2 \pi)$-realm, this identification corresponds to $e^{+i \pi}=e^{-i \pi}$ at the position where two Riemannian sheets are glued together. In this sense, the Dirac belt trick generalizes the construction of Riemannian sheets in one complex variable 
$z$ in the $(2 \pi)$-realm to the case of two complex variables $\left(Z_{1}, Z_{2}\right) \in C^{2}$ in the $(4 \pi)$-realm. Explicitly, the mapping $C^{2} \rightarrow C P^{1}$ can be expressed as $\left(Z_{1}, Z_{2}\right) \rightarrow\left(1, Z_{2} / Z_{1}\right)$ with:

$$
z=\frac{Z_{2}}{Z_{1}}=\tan \left[\frac{\theta}{2}\right] e^{i \phi}
$$

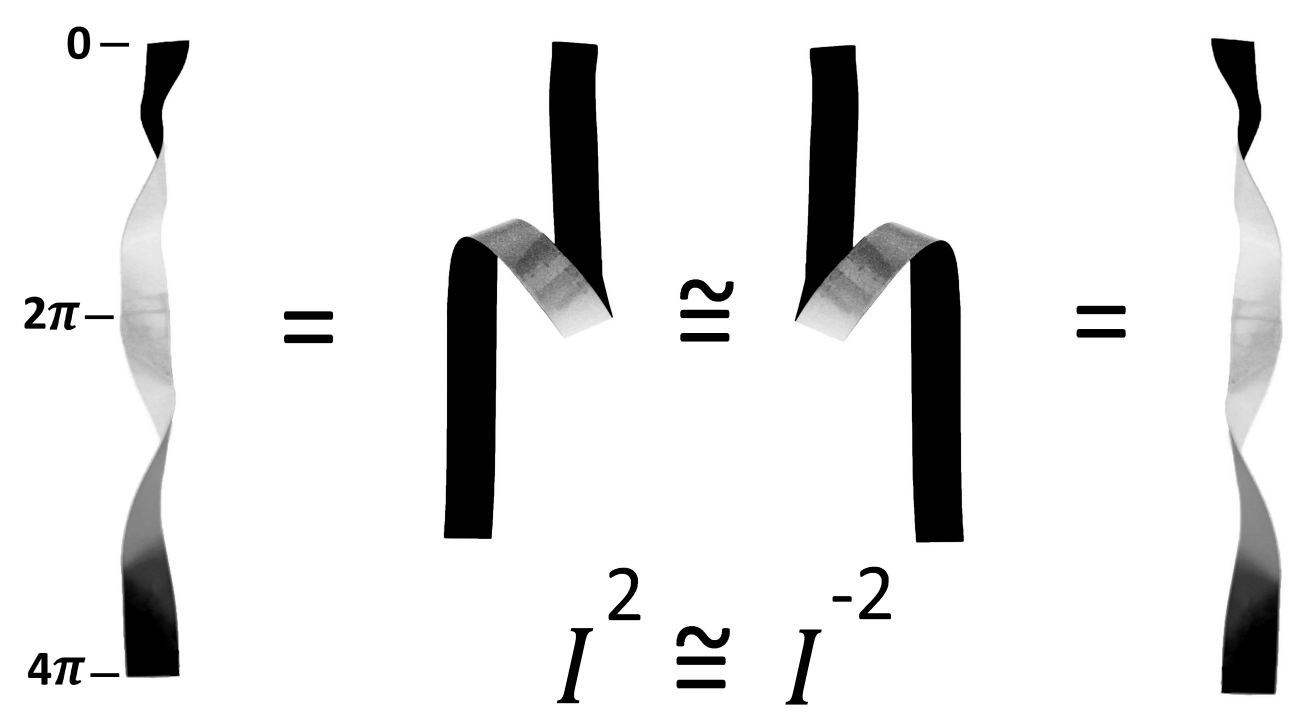

Figure 4. The operation $I^{2}=I^{-2}=-1$ introduces a single loop in the trivial state. The loop is equivalent to two clockwise twists $(++)$ or two counter-clockwise twists $(--)$. Note that twists in the $(4 \pi)$-realm (denoted \pm ) must carefully be distinguished from those in the $(2 \pi)$-realm (denoted $\mathrm{R} / \mathrm{L}$ ).

The coordinate $z$ can be mapped by stereographic projection onto the Bloch sphere parameterized with the usual coordinates of $S_{2}$, that is $\left(x_{1}=\cos [\phi] \sin [\theta], x_{2}=\sin [\phi] \sin [\theta], x_{3}=\cos [\theta]\right)$. The operator $U_{q}(\alpha)$ acting on the fundamental representation $\left(Z_{1}, Z_{2}\right)=(u, v)$ in $C^{2}$ (the $4 \pi$-realm) acts on the adjoint representation $X \equiv x_{i} \sigma_{i}$ on the Bloch sphere (the $2 \pi$-realm) as:

$$
U_{q}^{\dagger} X U_{q}=X^{\prime} .
$$

Explicitly, the rotation matrix $O\left(U_{q}\right) \in S O(3)$ acting on the vector $\left(x_{1}, x_{2}, x_{3}\right) \in S_{2}$ in the $(2 \pi)$-realm with $=x_{1}^{2}+x_{2}^{2}+x_{3}^{2}=1$ is given by:

$$
O_{k l}\left(U_{q}\right)=\frac{1}{\operatorname{tr}(\mathbf{1})} \operatorname{tr}\left(\sigma_{k} U_{q}^{\dagger} \sigma_{l} U_{q}\right) .
$$

The two-to-one mapping in the Hopf-mapping $S_{3} \rightarrow S_{2}$ becomes evident in this equation due to $O\left(U_{q}\right)=O\left(-U_{q}\right)$. Note that this equation holds for the case of general spin $j$, when $U$ is replaced by the corresponding $(2 j+1) \times(2 j+1)$ matrix representation of the rotation operator, and $\operatorname{tr}(\mathbf{1})=2 j+1$ (in terms of the coordinate $z \in C P^{1}$, this rotation is described by a Möbius transformation matrix $U_{q}$ ).

The two-to-one mapping can also be found in the paper strip model: Indeed, the Dirac belt representation in the $(4 \pi)$-realm can be mapped to the Möbius strip by gluing it together as shown in Figure 5. Inversely, by cutting the Möbius strip once in the middle, the Dirac belt with two surfaces emerges, where the phase is doubled from $2 \pi$ to $4 \pi$ (Figure 5). In such a way, we can extend the strip model from the $(2 \pi)$-realm, which is directly related to observables, to the $(4 \pi)$-realm, which is more abstract, but directly related to quantum phases in Hilbert space.

Next, let us generalize the Dirac belt to the case $j=1$ by applying the same operation to our paper strip representation of a state with two twists $T=+2,(R R)$ in the $(2 \pi)$-realm; see Figure 1 . The "doubling" of the bosonic state indeed is reproduced by the paper strip model, which decomposes into two identical copies of itself in the $(4 \pi)$-realm. Thus, in Hilbert space $C^{3}$, the phase of the bosonic 
state $j=1$ is described by two identical copies in a Hopf link; see Figure 5. Each copy has two twists, with $T^{\prime}=T \times T$ in the $(2 \pi) \times(2 \pi)$-realm; see Figures 2 and 6 . In this sense, bosons never explore the full $(4 \pi)$-realm, in contrast to fermions.
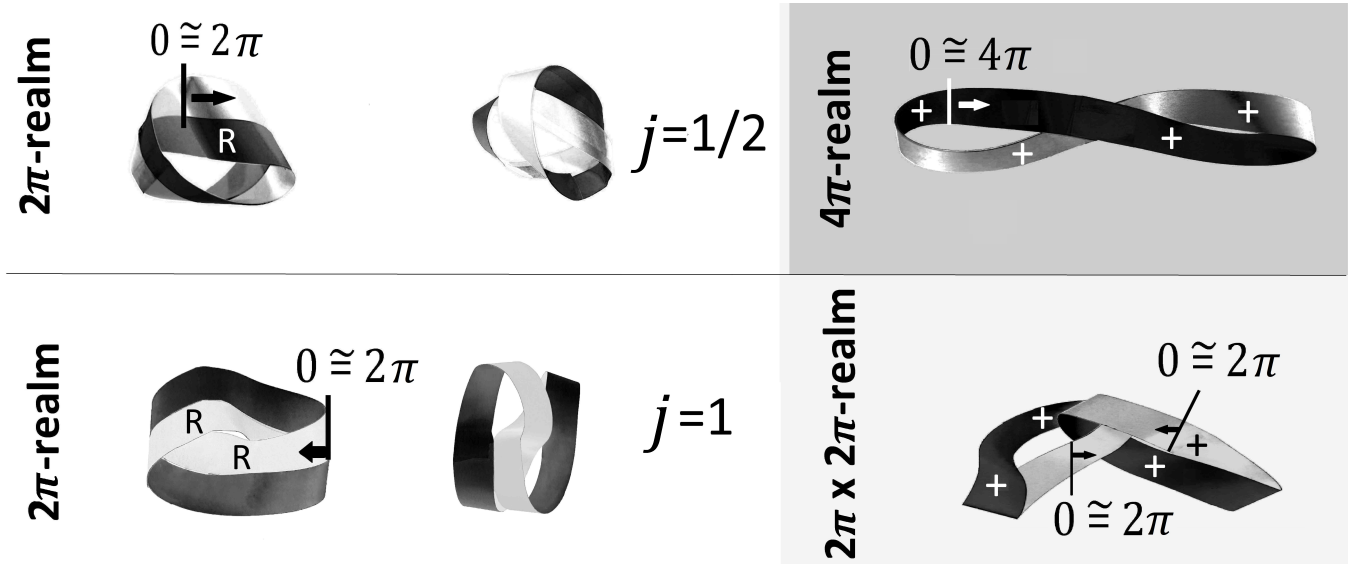

Figure 5. Upper row: Cutting the Möbius band $j=1 / 2$ (R in Figure 1) in the middle leads to a Dirac belt with four twists $T^{\prime}=4$, denoted by $(++++)$. Due to this operation, the length $2 \pi$ of the paper strip is extended to $4 \pi$, and the Möbius band-topology disappears. Lower row: The same operation for $j=1$ (RR in Figure 1) leads to two intertwined, identical copies of the original state (Hopf link), each again with two twists $T^{\prime}=T \times T$, in this case $(++) \times(++)$. The phase $2 \pi$ is not extended to $4 \pi$.

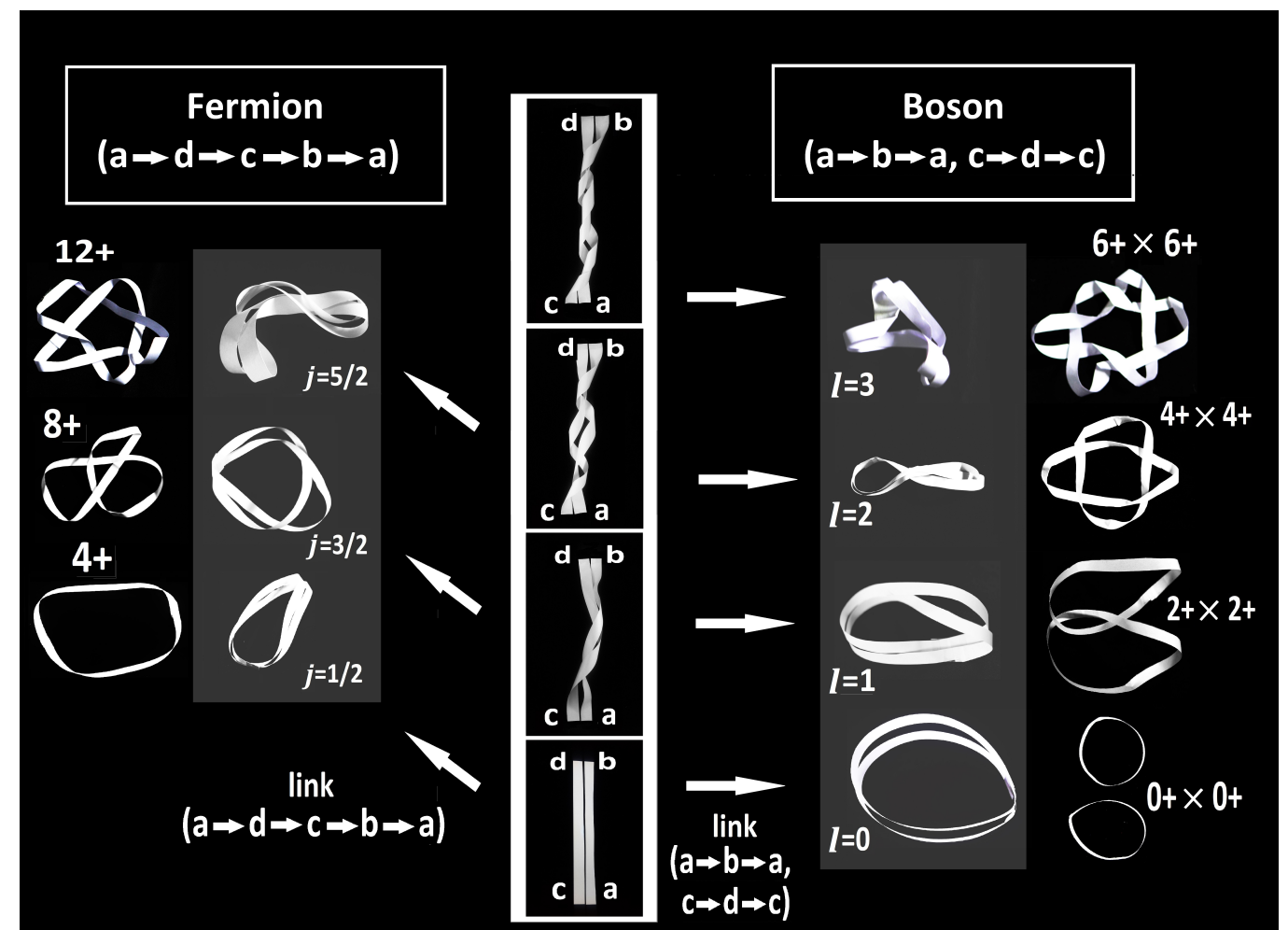

Figure 6. The paper strip visualization for spin $j=1 / 2, \ldots 3$ in the full Hilbert space, which is obtained from the model in the $(2 \pi)$-realm shown in Figure 1 by cutting the strip once lengthwise in the middle to double the phase. For fermions, a generalized Dirac belt emerges, described by a single knot. For bosons, two identical, intertwined copies of the original state shown in Figure 1 are obtained, leading to a generalized Hopf-link. Note that this is only possible for knots with inner twists $T^{\prime}=4 j+2$ or links with inner twists $T^{\prime}=2 j \times 2 j$ (here, $j=l$ must be integer). After Hopf-mapping, these twists are mapped to $2 j$ nodes in the stellar representation; see Figure 7. 


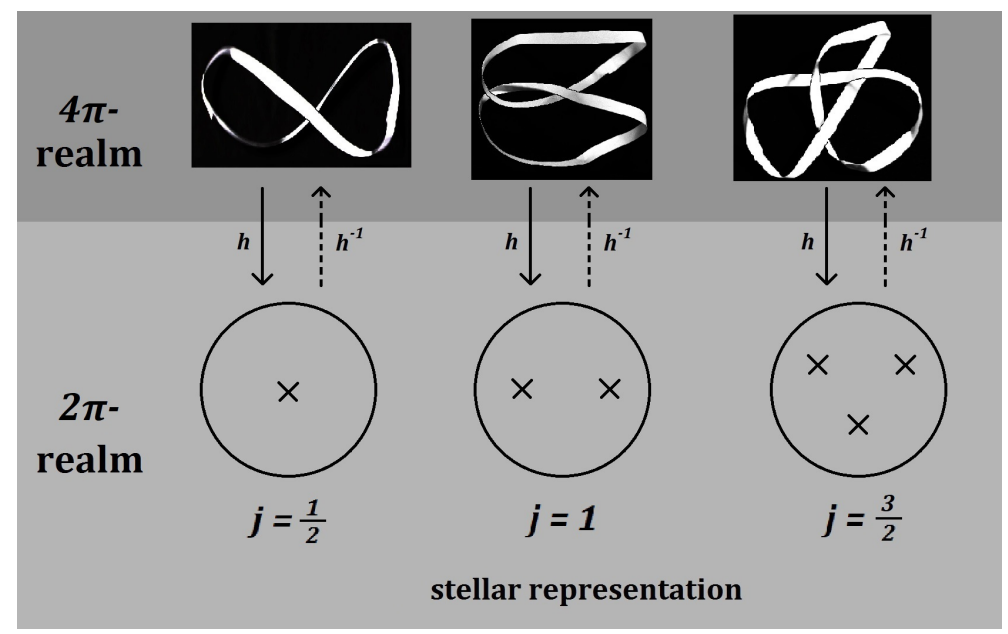

Figure 7. Relation between the stellar representation in the $(2 \pi)$-realm and of the the knot structure in the $(4 \pi)$-realm, illustrated for $j=1 / 2, j=1$ and $j=3 / 2$. Note that the number $p=2 j$ of nodes (sometimes called "stars") in the stellar representation on $S_{2}$ (corresponding to the number of twists Tin the paper strip model in the $(2 \pi)$-realm; see Figure 1$)$ determines the knot structure in the $(4 \pi)$-realm. The mapping $h$ is the Hopf mapping ( $h^{-1}$ being the inverse Hopf mapping). In Figure 6, the knot structure in the $(4 \pi)$-realm is represented in the paper strip model. The two-to-one Hopf mapping is achieved in the paper strip model by gluing the strips together, as shown in Figure 5.

\section{Relation Between Nodes, Twists and Knots}

Note that we have to distinguish carefully twists $T$ of the strip model in the $(2 \pi)$-realm and twists $T^{\prime}$ in the $(4 \pi)$-realm: twists $T$ in the $(2 \pi)$-realm are denoted by $R, L$ and can be observed as nodes of the quantum state. Twists $T^{\prime}$ in the $(4 \pi)$-realm are denoted by \pm . They are not directly related to observables. For this reason, twists in the $(4 \pi)$-realm are denoted as inner twists. Fermions with $j=1 / 2$ only appear to live on a Möbius strip with $T=1$, corresponding to one node. The antipode of the single node of the spin $j=1 / 2$ state defines the direction of the spin on the Bloch sphere. However, this is just our $(2 \pi)$-realm viewpoint of a states that indeed explores the full $(4 \pi)$-realm in the Hilbert space with $T^{\prime}=4$ inner twists in the quantum phase, which is nothing but the Dirac belt.

Mathematically, various approaches exist to describe states with spin $j$ both in the $(4 \pi)$-realm and in the $(2 \pi)$-realm. We may extend the Hilbert space $C^{2}$ for $j=\frac{1}{2}$ to $C^{2 j+1}$, introducing the homogeneous coordinates $Z_{1}, Z_{2}, \ldots Z_{2 j+1}$. For $S U(2)$-representations, the full hypersphere $S^{4 j+1}$ of possible quantum states is not explored (this would be the orbit of a pure state rotated by the full group $U(2 j+1)$ ). Rather, following Kramer [6,9], the submanifold where $S_{3}$ is wrapped $2 j$ times onto itself leads to a representation of the spin $j$ state in the $(4 \pi)$-realm, with homogeneous coordinates $Z_{k}$ $(k=1, \ldots 2 j+1)$ given by:

$$
Z_{k}=\left(\begin{array}{c}
2 j \\
k
\end{array}\right) 1 / 2 u^{k} v^{2 j-k}
$$

Indeed, $|Z|^{2}=\sum_{k=0}^{2 j} Z_{k}^{*} Z_{k}=\left(|u|^{2}+|v|^{2}\right)^{2 j}=1^{2 j}=1$ defines a (2j)-to-one mapping $S_{3} \rightarrow S_{3}$. Together with the operator $U_{q}$, the explicit form of the $(2 j+1) \times(2 j+1)$ representation of the generators $\left[J_{z}, J^{ \pm}\right]= \pm \hbar J^{ \pm},\left[J^{+}, J^{-}\right]=2 \hbar J_{z}$ of the group $\mathrm{SU}(2)$ can be deduced [9].

The Hopf-mapping from the $(4 \pi)$-realm $C^{2 j+1}$ to the $(2 \pi)$-realm $C P^{1}$ (equivalently, on $S_{2}$ ) can be done in various ways. The adjoint representation given in Equation (13) is one explicit example of the mapping on $S_{2}$. In such a way, the knot structure shown in Figure 6 is mapped to $p=2 j$ nodes on $S_{2}$. These nodes can be represented in the so-called stellar representation as zeros of a complex polynomial 
in one complex variable $z$ of order $p$, as first advocated by Majorana [6,10]. A similar approach is given by the co-called coherent state representation:

$$
|z\rangle=\frac{1}{\left(1+|z|^{2}\right)^{j}} e^{z J^{-}}|j, j\rangle=\frac{1}{\left(1+|z|^{2}\right)^{j}} \sum_{m=-j}^{m=+j}\left(\begin{array}{c}
2 j \\
j+m
\end{array}\right)^{1 / 2} z^{j+m}|j, m\rangle .
$$

Indeed, the polynomial in $z$ is of order $2 j$, leading to $p=2 j$ nodes, just as in the stellar representation. Note that the number of $p=2 j$ nodes exactly corresponds to the number of twists $T$ in the $(2 \pi)$-realm. Using the paper strip model, the relation between these nodes in $C P^{1}$ and the knot structure in $C^{2 j+1}$ is illuminated.

The paper strip model is a generalization of the Dirac belt construction to all spin states in the $(4 \pi)$-realm. Moreover, the two-to-one mapping of the quantum phase of a spin $j$-state from the Hilbert space in the $(4 \pi)$-realm to observables in the $(2 \pi)$-realm is made explicit by gluing together the paper strip, as shown in Figure 5. The number of twists $T$ coincides with the number of nodes in stellar representation, as shown in Figure 7 for $T=1$ and $T=2$. The generalization of this construction is shown in Figures 2 and 6. Note that our approach generalizes the model introduced in [3], since the two strands shown in Figure 2 are replaced by two strips shown in Figure 6, allowing for inner twists even in the $(4 \pi)$-realm and thus giving a description of the quantum phase in Hilbert space. As shown in Figure 5, the state $j=1 / 2$ is described as the Dirac belt with four twists $T^{\prime}=4,(++++)$. As is obvious from the construction shown in Figure 2, if the two strands are replaced by two strips, each strip in the braid has $2 l$ twists. For bosons, this leads to $T^{\prime}=2 l \times 2 l$ inner twists in the $(2 \pi) \times(2 \pi)$-realm. For the corresponding fermion with $j=l+1 / 2$, four inner twists are added when the strips are connected, leading to $T^{\prime}=4 l+4=4 j+2$ inner twists in the $(4 \pi)$-realm, as shown in Figure 6 . The number of inner twists $T^{\prime}$ in the $(4 \pi)$-realm or the $(2 \pi) \times(2 \pi)$-realm has to adhere to these rules to map to an observable state with $T=2 j$ twists (equivalently, $2 j$ nodes) in the $(2 \pi)$-realm.

\section{Observable Effects of the Möbius-Strip Topology}

In the $(2 \pi)$-realm, a rotation by $\theta=2 \pi$ leads for bosons back to the original state. For fermions, however, this rotation leads to a sign change.

The sign change can be directly measured from the interference patterns of fermions (i.e., as a relative phase) [11,12].

As can be seen in Figure 1, the Möbius strip-like topology for half-integer $j$ (the number of twists $p=2 j$ is odd in this case) illustrates the double-valuedness of the spinor wave function, as one has to travel along the strip for $4 \pi$ to get back to the original point.

\section{Summary and Outlook}

The paper strip model introduced in Figure 6 naturally leads to knot theory, revealing the knot-theoretic origin of the boson-fermion correspondence [8]. The dramatic difference between bosons and fermions becomes relevant only after Hopf-mapping to the $(2 \pi)$-realm. It is crucial to distinguish carefully the $(2 \pi)$-realm $S_{2}$ (equivalently, $C P^{1}$ ) from the $(4 \pi)$-realm $S_{3}$ (more generally, $\left.C^{2 j+1}\right)$ for the description of quantum states. Spin- $-\frac{1}{2}$ states are described by the spinor $(u, v)$ in the $(4 \pi)$-realm, but the corresponding observables are expressed on the Bloch sphere or by $z=u / v \in C P^{1}$ after Hopf-mapping to the $(2 \pi)$-realm. The two-to one mapping leads to a Möbius band topology for fermions; see Figure 5.

Bosonic states are usually described in the $(2 \pi)$-realm ignoring the identical copy of the state in the $(2 \pi) \times(2 \pi)$-realm. Note that Artin's braid group describing bosonic and fermionic states as shown in Figure 2 is extended by the strip model, since not only crossings and self-crossings have to be taken into account, but also inner twists of each of the two paper strips, as shown in Figure 6, which gives another prerequisite for $(4 \pi)$-realm and $(2 \pi) \times(2 \pi)$-realm states to be observable in the $(2 \pi)$-realm. 
In such a way, our model generalizes the Dirac belt [1] and also the rubber-band model introduced by Finkelstein and Rubinstein [13], which both describe quantum phases in Hilbert space, that is, in the $(4 \pi)$-realm before Hopf-mapping. Moreover, our model clarifies and generalizes the relation of the model for spin- $\frac{1}{2}$ particles in the $(2 \pi)$-realm introduced by J.Avrin [3] and the (generalized) Dirac belt in the $(4 \pi)$-realm. In contrast to Avrin, our model allows for inner twists $T^{\prime}$ in the $(4 \pi)$-realm. Crucially, the mechanism of the two-to-one Hopf mapping from quantum phases in Hilbert space in the $(4 \pi)$-realm to observables in the $(2 \pi)$-realm is illuminated by gluing together the knot, modeled as a paper strip with $T^{\prime}=4 j+2$ inner twists and a knot structure described by the Jones polynomial $J^{j}(t)(9)$, as shown in Figures 5 and 6. The result of the mapping is a Möbius strip with $T$ twists in the $(2 \pi)$-realm. For bosons with integer $j=l$, the link modeled as a paper strip with $T^{\prime}=2 l \times 2 l$ inner twists is mapped to itself, identifying both linked copies of the quantum state (in the model, this is achieved by gluing together both identical pieces of the link, as shown in Figure 5 for $l=1$ ). For bosons and for fermions, the number of twists $T$ in the $(2 \pi)$-realm coincides with the number of nodes in stellar representation, as shown in Figure 7 for $T=1,2,3$.

Applications of the paper strip model and its underlying knot theoretical approach are possible, e.g., for the description of topological charges in gauge theories, for the spin-statistics theorem, and for entanglement, as will be shown elsewhere.

It must also be emphasized that the paper strip model might have merit in physics education due to its haptic nature, allowing one to experience an abstract and important concept in mathematical physics on a qualitative level.

Author Contributions: Both authors equally contributed to the paper.

Funding: This research received no external funding.

Conflicts of Interest: The authors declare no conflict of interest.

\section{References}

1. Staley, M. Understanding Quaternions and the Dirac Belt Trick. Eur. J. Phys. 2010, 31, 467-478. [CrossRef] [PubMed]

2. Heusler, S.; Ubben, M. Modelling spin. Euro. J. Phys. 2018, 39, 065405. [CrossRef]

3. Avrin, J. Knots on a Torus: A model of the Elementary particles. Symmetry 2012, 4, 39-115. [CrossRef]

4. Milnor, J. Singular Points of Complex Hypersurfaces; Princton University Press: Princton, NJ, USA, 1928.

5. Brauner, K. Zur Geometrie der Funktionen zweier komplexer Veränderlichen. Abh. Math. Semin. Hambg. 1928, 6, 1-54. [CrossRef]

6. Bengtsson, I.; Zyczkowski, K. Geometry of Quantum States; Cambridge University Press: Camebridge, UK, 2006.

7. Kauffman, L. The mathematics and physics of knots. Rep. Prog. Phys. 2005, 68, 2829. [CrossRef]

8. Frenkel, I.; Penkov, I.; Serganova, V. A Categorification of the Boson-Fermion Correspondence via Representation Theory of sl( $\infty)$. Commun. Math. Phys. 2016, 341. [CrossRef]

9. Brinkman, H.C. Applications of Spinor Invariants in Atomic Physics; North Holland: Amsterdam, The Netherlands, 1956.

10. Majorana, E. Atomi orientati in campo magnetico variabile. Nuovo Cimento 1932, 9, 43-50. [CrossRef]

11. Bernstein, H. Spin precession during interferometry of fermions and the phase factor associated with rotations through 2p radians. Phys. Rev. Lett. 1967, 18, 1102-1103. [CrossRef]

12. Werner, S.; Colella, R.; Overhauser, A.; Eagen, C. Observation of the phase shift of a neutron due to precession in a magnetic field. Phys. Rev. Lett. 1975, 35, 1053-1055. [CrossRef]

13. Finkelstein, D.; Rubinstein, J. Connection between spin, statistics, and kinks. J. Math. Phys. 1968, 9, 1762-1779. [CrossRef]

(C) 2019 by the authors. Licensee MDPI, Basel, Switzerland. This article is an open access article distributed under the terms and conditions of the Creative Commons Attribution (CC BY) license (http://creativecommons.org/licenses/by/4.0/). 